\title{
Constructing Fund of Funds Portfolio with Risk Parity
}

\author{
Jianlin Zhao ${ }^{\text {a }}$, Ming Zhou ${ }^{\text {b }}$ \\ Central University of Finance and Economics, Beijing 100081, China; \\ azhaojianlin@aliyun.com, brhouming@cufe.edu.cn
}

Keywords: risk parity; portfolio; risk budgeting; optimization.

\begin{abstract}
Risk parity became a popular financial model of investment after the global financial crisis in 2008. Today, pension funds and institutional investors are using this approach in the development of smart indexing and the redefinition of long-term investment policies. Introduction to Risk Parity and Budgeting provides an up-to-date treatment of this alternative method to Markowitz optimization. It builds financial exposure to equities and commodities, considers credit risk in the management of bond portfolios, and designs long-term investment policy. In this paper, a risk parity portfolio constructed using three large assets, CSI Total Debt Index, Wind A Index and South China Commodity Index.
\end{abstract}

\section{Introduction}

Since the mean-variance portfolio optimization framework was introduced by Markowitz over fifty years ago [1-3], it has been well-researched in the academic field. However, practitioners have not embraced such a nice theoretical framework. There are several reasons. The first reason is that this approach relies on the estimates of expected returns and the obtained portfolio is highly unstable. This may be overcome partially by introducing additional constraints on the portfolio. Another severe drawback of the Markowitz portfolio is that the approach tends to provide an excessively concentrated portfolio and risk over a few assets, which goes against the common sense of diversification as a way to reduce the risk. During normal times this may not cause a serious issue, but if a financial crisis were to happen, such a concentrated portfolio would probably incur huge losses.

The new paradigm of risk parity portfolio was precisely introduced to make the portfolio, and hence the risk, truly more diversified. Qian [4-5] first showed that uniform risk contributions actually lead to a diverse enough portfolio, and the (ex-ante)b risk contributions(i.e., the risk computed suing historical data) are not only a mathematical measurement of how diverse the risk is, but also good indicators of the (ex-post) loss contributions of the assets (i.e., the observed risks and losses is to distribute the risk in the future), especially when exist large losses. According to this observation, the way to avoid a potential huge loss is to distribute the risk contributions. This method has been receiving significant attention recently, especially after the 2008 financial crisis, and it has been shown that a risk parity portfolio is also more robust than the Markowitz portfolio.

This paper is organized as follows. Section 2 introduces the risk parity theory and a risk parity portfolio constructed in section 3. Finally, a summary of this paper will be written in section 4 .

\section{Risk Parity}

\subsection{Risk Contribution}

Suppose there are $n$ assets with random returns $r \in \mathbb{R}^{n}$, and the mean vector and (positive definite) covariance matrix are denoted as $\boldsymbol{\mu} \in \mathbb{R}^{n}$ and $\boldsymbol{\Sigma} \in \mathbb{R}^{n \times n}$. We use $\mathbf{w} \in \mathbb{R}^{n}$ to denote the normalized portfolio (e.g., $\mathbf{w}^{T} \mathbf{1}=1$ ), which describes how the total capital budget it to be allocated over the assets. To study the risk parity portfolio, we need some well-defined risk measurement

$$
f(\mathbf{w})=\sum_{i=1}^{n} \omega_{i} \frac{\partial f}{\partial \omega_{i}}
$$

so that the "risk contribution" of each asset to the risk of the whole portfolio can be quantified. The following desired property is always introduced in risk parity literature. 


\subsection{Risk Measurement}

Note that variance $\sigma^{2}(\mathbf{w})=\mathbf{w}^{T} \boldsymbol{\Sigma} \mathbf{w}$ does not satisfy (1) directly. Fortunately, it is easy to check that volatility $\sigma(\mathbf{w})=\sqrt{\mathbf{w}^{T} \mathbf{\Sigma} \mathbf{w}}$ does satisfy (1)

$$
\sum_{i=1}^{n} \omega_{i} \frac{\partial \sigma}{\partial \omega_{i}}=\sum_{i=1}^{n} \omega_{i}\left(\frac{\mathbf{\Sigma} \mathbf{w}}{\sqrt{\mathbf{w}^{T} \mathbf{\Sigma} \mathbf{w}}}\right)_{i}=\frac{1}{\sqrt{\mathbf{w}^{T} \mathbf{\Sigma} \mathbf{w}}} \sum_{i=1}^{n} \omega_{i}(\mathbf{\Sigma} \mathbf{w})_{i}=\frac{1}{\sqrt{\mathbf{w}^{T} \mathbf{\Sigma} \mathbf{w}}} \mathbf{w}^{T} \mathbf{\Sigma} \mathbf{w}=\sigma(\mathbf{w})
$$

Thus variance fits (1) indirectly via volatility.

\subsection{Risk Parity/Budgeting Portfolio}

This risk parity portfolio is a portfolio such that each asset has the same risk contribution. That is, given the risk measurement $f(\mathbf{w})$, the risk parity portfolio should satisfy

$$
\omega_{i} \frac{\partial f(\mathbf{w})}{\partial \omega_{i}}=\omega_{j} \frac{\partial f(\mathbf{w})}{\partial \omega_{j}}, \quad \forall i, j
$$

Risk budgeting portfolio is a more general concept. Given a budget vector $\mathbf{b}=\left[b_{1}, \ldots, b_{n}\right]^{T}>0$, and $\mathbf{b}^{T} \mathbf{1}=1$, where budget $\mathbf{b}$ is interpreted as a pre-determined percentage risk contribution target for all the assets, the risk budgeting portfolio should satisfy

$$
\omega_{i} \frac{\partial f(\mathbf{w})}{\partial \omega_{i}}=b_{i} f(\mathbf{w}), \quad \forall i
$$

Obviously, the risk parity portfolio is a special case of the risk budgeting portfolio with $\mathbf{b}=\frac{\mathbf{1}}{n}$.

Due to the popularity of the terminology "risk parity", for clarity of presentation, we mainly refer "risk parity" as a broad portfolio allocation method of risk diversification (e.g., including both risk parity and risk budgeting portfolios) unless specified otherwise in this paper.

\section{Back-testing}

In this section, a risk parity portfolio constructed using three large assets, CSI Total Debt Index, Wind A Index and South China Commodity Index. The three categories of assets are selected from January 2, 2005 to June 3, 2017. Their performance trend shown in Figure 1.

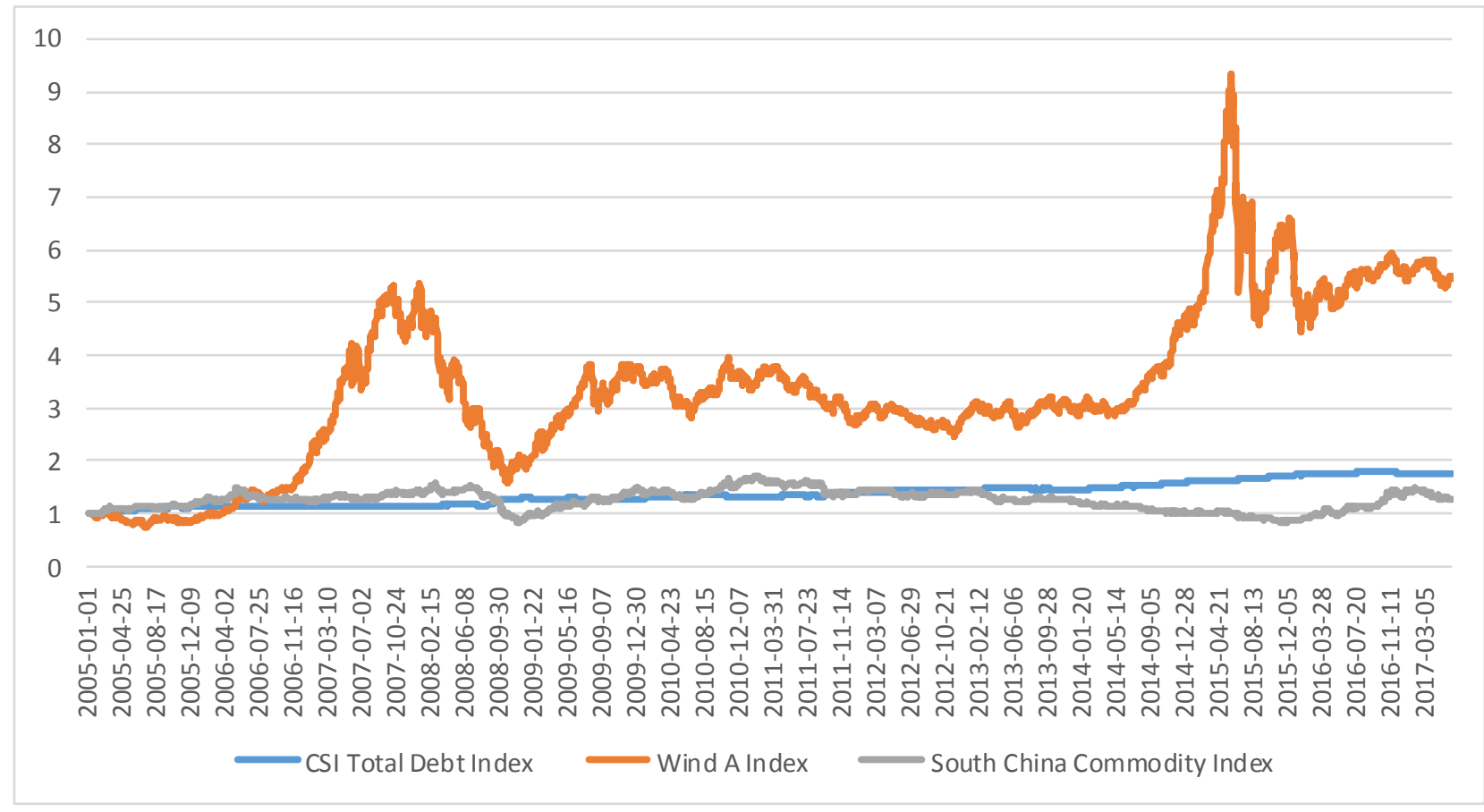

\subsection{Correlation analysis}

Fig. 1 Three categories of assets' performance trend

The prerequisite of risk parity theory is that there is a low correlation between assets. Therefore, it is necessary to first calculate whether the correlation between the selected assets meets the requirements. 
Table 1. Correlation of three assets (CSI Total Debt Index, Wind A Index and South China Commodity Index)

\begin{tabular}{cccc}
\hline & CSI Total Debt Index & Wind A Index & South China Commodity Index \\
\hline CSI Total Debt Index & 1.00 & -0.02 & -0.05 \\
Wind A Index & & 1.00 & 0.31 \\
South China Commodity Index & & & 1.00 \\
\hline
\end{tabular}

As can be seen from Table 1, there is a low correlation between assets, so you can use these assets to construct a risk parity combination.

\subsection{Risk Parity Portfolio Construction and Comparison with other Portfolios}

In order to highlight the combination of risk parity, we compared the combination of risk parity and equal weighted portfolio. The configuration results shown in Figure 2.

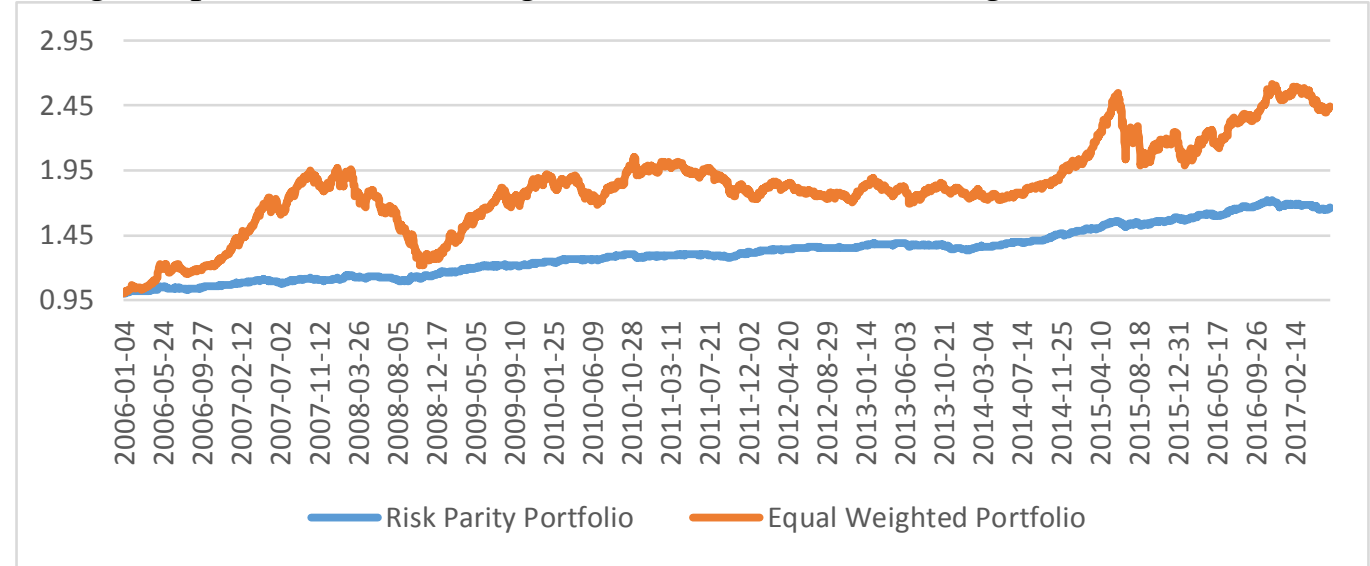

Fig. 2 The trend of risk parity portfolio and equal weighted portfolio

The quantification of these two combinations is shown in Table 2. As can be seen from Table 2, the risk parity combination has a greater Sharp ratio and a larger Calmar ratio. Therefore, if the combination of risk parity to increase the leverage, then bear the same risk in the case, you can get a higher weight ratio than the combination of weight.

Table 2. Quantitative indicators of two portfolios

\begin{tabular}{cccccc}
\hline & $\begin{array}{c}\text { Annualized rate of } \\
\text { return }\end{array}$ & $\begin{array}{c}\text { Annualized } \\
\text { volatility }\end{array}$ & $\begin{array}{c}\text { Maximum } \\
\text { retracement }\end{array}$ & $\begin{array}{c}\text { Sharp } \\
\text { ratio }\end{array}$ & $\begin{array}{c}\text { Calmar } \\
\text { ratio }\end{array}$ \\
\hline $\begin{array}{c}\text { Risk parity } \\
\text { portfolio }\end{array}$ & $4.69 \%$ & $2.85 \%$ & $4.43 \%$ & 1.65 & 1.06 \\
$\begin{array}{c}\text { Equal weighted } \\
\text { portfolio }\end{array}$ & $8.41 \%$ & $12.80 \%$ & $38.01 \%$ & 0.66 & 0.22 \\
\hline
\end{tabular}

\section{Summary}

In this section, the advantages and the dis-advantages of the risk parity portfolio will be included as follows:

Firstly, let us summarize the advantages of risk parity theory as follows:

1. It doesn't need to predict future and the future is also uncertain;

2. It puts different assets together to really achieve diversified the risk;

3. It pursues the absolute return;

4. It can avoid performance trap.

Secondly, the dis-advantages of the risk parity portfolio are as follows:

1. The existing risk parity strategies are volatility parities, not real risk parities, from the point of view of volatility. But risk is not volatility. Risk is essentially a possibility of a permanent loss, and volatility is only a way of describing risk. For example, default risk is difficult to describe volatility.

2. The tail risk is not considered: most assets have a thick tail and do not obey the normal distribution. The risk parity strategy gives the same standard difference weights for all assets. When the standard deviation of investment grade corporate bonds and stocks has the same 
weight, the tail risk of investment grade corporate bonds may be 4 times that of stocks, which greatly increases the tail risk of the whole portfolio. Therefore, the excess return of the risk parity strategy is higher than that of other investment strategies, possibly because the risk parity strategy increases the tail risk.

3. Non reaction investor psychology: it is obvious that investors' negative feelings of loss are far stronger than the positive feelings of profits. In the risk parity strategy, asset price increases and price falls have equal risks. However, investors do not equate the risk of price increases with the risk of falling prices. The risk parity strategy does not reflect the investor's true view of their portfolio.

\section{References}

[1]. Markowitz H. Portfolio selection [J]. The journal of finance, 1952, 7(1): 77-91.

[2]. Markowitz H. The optimization of a quadratic function subject to linear constraints [J]. Naval research logistics Quarterly, 1956, 3(1 - 2): 111-133.

[3]. Markowitz H M. Portfolio selection: efficient diversification of investments [M]. Yale university press, 1968.

[4]. Qian E. Risk parity portfolios: Efficient portfolios through true diversification [J]. Panagora Asset Management, 2005.

[5]. Qian E E. On the financial interpretation of risk contribution: Risk budgets do add up [J]. 2005. 